\title{
28 Research Suare \\ Characterization of PVPK90 core shell nanofibers containing curcumin prepared by emulsion electrospinning process
}

\section{Hossein Kamali}

Mashhad University of Medical Sciences

\section{Parisa Farzadnia}

Mashhad University of Medical Sciences

Jebraeil Movaffagh

Mashhad University of Medical Sciences

Mohammadreza Abbaspour ( $\sim$ abbaspourmr@mums.ac.ir)

Mashhad University of Medical Sciences

\section{Research Article}

Keywords: Curcumin, Emulsion-electrospinning, nanofibers, PVPK90, O/W emulsion

Posted Date: June 22nd, 2021

DOI: https://doi.org/10.21203/rs.3.rs-618802/v1

License: (c) (i) This work is licensed under a Creative Commons Attribution 4.0 International License. Read Full License 


\section{Abstract}

The pharmacological effects of curcumin (CUR) as a polyphenolic ingredient of turmeric affected by its water insolubility, poor bioavailability, and instability. The electrospun nanofibers of hydrophilic biodegradable polymer can be addressed this issue. The current study aimed to investigate the CUR-loaded core-shell PVPK90 nanofibers generated from O/W nanoemulsions. CUR-loaded PVPK90 fibers were prepared based on the central composite design and different levels of drug concentration, flow rate, amount of acetone, and organic phase percentage by electrospinning technique and their diameter and uniformity, tensile strength, wetting and disintegration time were evaluated. Then, DSC, FTIR, XRD, TEM, fluorescence microscopy, and drug release test were carried out on the optimized nanofibers. The formulation containing $6 \mathrm{mg} / \mathrm{mL}$ CUR, $30 \%$ organic phase, $0.4 \mathrm{~mL}$ acetone, and flow rate of $1 \mathrm{~mL} / \mathrm{h}$ was selected as optimum formulation. The results were confirmed the core-shell bead-free uniform fibers at the nanometer scale. The optimum nanofiber showed a good flexibility, short wetting and disintegrating times as well as the amorphous structure. The drug release pattern indicated the rapid release of the drug within $30 \mathrm{~min}$. Our findings confirm that the $\mathrm{O} / \mathrm{W}$ emulsion electrospinning-based PVPK90 core-shell nanofiber would be beneficial as a novel delivery system for water insoluble drug.

\section{Introduction}

Curcumin (CUR), a low molecular weight natural polyphenol, is an FDA-approved molecule isolating from the rhizome of the Curcuma longa and is usually used as a food additive ${ }^{1,2}$. CUR displays antioxidant, anti-inflammatory, and antitumor properties and involves in a wide range of pharmacological activities. It also is helpful in various diseases including infection, cancer, Alzheimer, diabetes, and cardiovascular diseases ${ }^{1,3,4}$. The low solubility in aqueous media, physicochemical instability, fast systemic elimination, low permeability, and low bioavailability are some negative properties of CUR ${ }^{5-7}$. Some approaches for instance the conjugation of CUR with the biodegradable polymer led to improved water solubility, increased blood stability and time-circulation, enhanced permeability and bioavailability, and resistance to depletion ${ }^{8,9}$. The polymeric nanofibers as a nanoscale structure display a great potential for the application of CUR in the aqueous phase because of the large surface area, tunable porosity, degradability, and ease of functionalization which are facilitated the efficient CUR delivery ${ }^{10,11}$

Among the nanofiber production methods, the electrospinning technique is one of the most favorable, simple, and inexpensive tools for the construction of polymeric nanofibers from natural or synthetic polymers ${ }^{12}$. In this system, by applying the high-voltage electric supply, a polymer melt or solution is pumped through a spinneret at a certain flow rate resulting in the excretion of a charged polymer jet from a capillary which is accelerated towards the collector. During the collection of fiber jet on a grounded target, the solvent evaporates and the solid or coagulated ultrafine nanoscale fibers (nanofibers) are generated ${ }^{13,14}$. Recently, the electrospun nanofibers consider proper materials for widespread applications such as healthcare, environmental engineering, nanotextiles, as well as biomedical fields e.g. wound healing materials, filtration membranes, regenerative medicine, tissue engineering, and drug delivery ${ }^{15-17}$. The emulsion electrospinning, as a commonly used electro-spinning method, involves a water-in-oil (W/O) or oil-in-water (O/W) emulsions, which is constructed the core-shell nanofibers encapsulated with hydrophilic or hydrophobic compounds ${ }^{18}$. In comparison with coaxial electrospinning, emulsion-based spinning is a simple technique for core/shell nanofibers fabrication which is effective for loading the material that is only dissolved in an organic solvent into a aqueous media or vis versa ${ }^{19}$. Moreover, unlike to coaxial method, the core-shell fibers formation is based on single nozzle and single solution in the emulsion electrospinning ${ }^{20,21}$. Due to the low toxicity, biodegradability, and biocompatibility of polymers that are used in emulsion-based electrospinning, these polymers-produced nanofibers have attracted a lot of attention for nanomedicine ${ }^{18,22}$. 
One of the polymers that is extensively used for nanofibers production is Polyvinylpyrrolidone (polyvidone/PVP) which is improved the solubility of some hydrophobic drugs/components ${ }^{23}$. PVP is a linear commercial nonionic, hygroscopic, amorphous, hydrophilic vinyl polymer made from the monomer $\mathrm{N}$-vinyl-pyrrolidone ${ }^{24,25}$. PVP is available in varied types like homopolymers with diverse grades depend on its average molecular weight, copolymers, and crosslinked forms ${ }^{26}$. PVP can be applied in the numerous fields for instance cosmetic and food industry, pharmaceutical, and biomedical concerns owing to their proper water solubility, affinity to different hydrophilic or hydrophobic drugs, adhesiveness to various substrates, and good chelate/complex formation hallmark ${ }^{24,25}$. It is used as an emulsion stabilizer, hair fixative agent, and particularly as an excipient in many pharmaceutical tablets, and involves in controlled drug delivery 25,26 .

In current study, we define a new one-step process to achieve core-shell hydrophilic CUR-loaded PVPK90 nanofibers based on O/W nanoemulsion electrospinning based on dissolving the PVP in the continuous phase of the nanoemulsion. The central composite design was used to assess the formulations based on the main variable including the CUR concentration, flow rate, amount of acetone, and \% organic phase. Then, the optimum formulation was selected based on the evaluation of the diameter, uniformity, flexibility, drug content, wetting, and disintegration time of fibers. The optimized nanofiber properties were assayed by DSC, FTIR, XRD, TEM, and fluorescence microscopy, and its drug release pattern was determined.

\section{Results And Discussion}

\section{Correlation between process parameters and fiber diameter}

In our preliminary studies, PVPK30 (40,50, and 60\% wt) and PVPK90 are used to fabricate nanofibers. The PVPK30-based solutions formed electro sprayed beads instead of nanofibers. On the contrary, bead-free electrospun fibers were produced from PVPK90 due to their higher viscosity compare to PVPK30. It is demonstrated that the high viscosity of the electrospinning solution causes less bead formation in spunfibers ${ }^{27,28}$. To achieve the optimal formulation for making electrospun nanofibers containing CUR with PVPK90 and to check the effect of variables on the studied responses, 21 formulations by central composite design with 5 center points were determined. Formulations 11 and 13 cannot be achieved because CUR did not dissolve in the organic phase, completely (Table 1). 
Table 1

The results of nanofiber evaluation tests (Fiber diameter, entrapment percentage, wetting and disintegration time) in different formulation

\begin{tabular}{|c|c|c|c|c|c|c|c|c|}
\hline Formulation & $\begin{array}{l}X_{1}=A \\
\begin{array}{l}\text { Acetone } \\
(\mathrm{ml})\end{array}\end{array}$ & $\begin{array}{l}\mathrm{X}_{2}=\mathrm{B} \\
\text { Flow } \\
\text { rate } \\
(\mathrm{ml} / \mathrm{h})\end{array}$ & $\begin{array}{l}X_{3}=C \\
\text { Organic } \\
\text { phase } \\
(\%)\end{array}$ & $\begin{array}{l}X_{4}=D \\
\text { Curcumin } \\
\text { concentration } \\
(\mathrm{mg} / \mathrm{mL})\end{array}$ & $\begin{array}{l}\text { Fiber } \\
\text { Diameter } \\
(\mathrm{nm})\end{array}$ & $\begin{array}{l}\text { Entrapment } \\
(\%)\end{array}$ & $\begin{array}{l}\text { Wetting } \\
\text { time } \\
\text { (sec) }\end{array}$ & $\begin{array}{l}\text { Disintegration } \\
\text { Time } \\
\text { (sec) }\end{array}$ \\
\hline \multirow[t]{2}{*}{1} & 0.3 & 0.7 & 25 & 10 & $909.97 \pm$ & $48.08 \pm$ & $2.76 \pm$ & $37.33 \pm$ \\
\hline & & & & & 127.74 & 4.12 & 0.76 & 5.68 \\
\hline \multirow[t]{2}{*}{2} & 0.2 & 0.7 & 25 & 8 & $\begin{array}{l}1171.02 \\
\pm\end{array}$ & & & \\
\hline & & & & & & 2.33 & 0.93 & 3.05 \\
\hline \multirow[t]{2}{*}{3} & 0.3 & 0.4 & 25 & 8 & $948.47 \pm$ & $33.60 \pm$ & $2.3 \pm$ & $32 \pm$ \\
\hline & & & & & 169.99 & 6.83 & 0.85 & 6.24 \\
\hline \multirow[t]{2}{*}{4} & 0.3 & 1 & 25 & 8 & $729.11 \pm$ & $33.65 \pm$ & $3.96 \pm$ & $66.33 \pm$ \\
\hline & & & & & 92.62 & 0.59 & 0.15 & 9.29 \\
\hline \multirow[t]{2}{*}{5} & 0.3 & 0.7 & 25 & 8 & $799.56 \pm$ & $31.65 \pm$ & $2.76 \pm$ & $24.5 \pm$ \\
\hline & & & & & 111.96 & 3.35 & 0.35 & 2.33 \\
\hline \multirow[t]{2}{*}{6} & 0.3 & 0.7 & 25 & 6 & $747.97 \pm$ & $44.31 \pm$ & $3.53 \pm$ & $49.33 \pm$ \\
\hline & & & & & 97.99 & 5.06 & 0.28 & 5.85 \\
\hline \multirow[t]{2}{*}{7} & 0.3 & 0.7 & 25 & 8 & $799.56 \pm$ & $31.64 \pm$ & $2.8 \pm$ & $23.66 \pm$ \\
\hline & & & & & 113.95 & 2.33 & 0.53 & 2.08 \\
\hline \multirow[t]{2}{*}{8} & 0.3 & 0.7 & 25 & 8 & $799.60 \pm$ & $31.66 \pm$ & $2.75 \pm$ & $23.8 \pm$ \\
\hline & & & & & 110.46 & 2.65 & 0.37 & 2.28 \\
\hline \multirow[t]{2}{*}{9} & 0.3 & 0.7 & 25 & 8 & $780.10 \pm$ & $31.71 \pm$ & $2.74 \pm$ & $24 \pm$ \\
\hline & & & & & 113.34 & 2.30 & 0.23 & 2.58 \\
\hline \multirow[t]{2}{*}{10} & 0.3 & 0.7 & 25 & 8 & $781.32 \pm$ & $31.76 \pm$ & $2.73 \pm$ & $23.90 \pm$ \\
\hline & & & & & 119.25 & 2.46 & 0.34 & 2.67 \\
\hline 11 & 0.2 & 1 & 30 & 10 & * & * & * & * \\
\hline \multirow[t]{2}{*}{12} & 0.4 & 0.4 & 30 & 10 & $\begin{array}{l}1152.87 \\
\pm\end{array}$ & $48.13 \pm$ & $2.66 \pm$ & $49.66 \pm$ \\
\hline & & & & & & 3.37 & 0.65 & 2.51 \\
\hline 13 & 0.2 & 0.4 & 30 & 6 & * & $\star$ & $\star$ & * \\
\hline \multirow[t]{2}{*}{14} & 0.2 & 1 & 20 & 10 & $770.52 \pm$ & $51.2 \pm$ & $3.8 \pm$ & $72.66 \pm$ \\
\hline & & & & & 173.11 & 6.18 & 0.98 & 11.23 \\
\hline
\end{tabular}




\begin{tabular}{|c|c|c|c|c|c|c|c|c|}
\hline Formulation & $\begin{array}{l}X_{1}=A \\
\begin{array}{l}\text { Acetone } \\
(\mathrm{ml})\end{array}\end{array}$ & $\begin{array}{l}X_{2}=B \\
\text { Flow } \\
\text { rate } \\
(\mathrm{ml} / \mathrm{h})\end{array}$ & $\begin{array}{l}X_{3}=C \\
\text { Organic } \\
\text { phase } \\
(\%)\end{array}$ & $\begin{array}{l}\mathrm{X}_{4}=\mathrm{D} \\
\text { Curcumin } \\
\text { concentration } \\
(\mathrm{mg} / \mathrm{mL})\end{array}$ & $\begin{array}{l}\text { Fiber } \\
\text { Diameter } \\
(\mathrm{nm})\end{array}$ & $\begin{array}{l}\text { Entrapment } \\
\text { (\%) }\end{array}$ & $\begin{array}{l}\text { Wetting } \\
\text { time } \\
\text { (sec) }\end{array}$ & $\begin{array}{l}\text { Disintegration } \\
\text { Time } \\
\text { (sec) }\end{array}$ \\
\hline 15 & 0.4 & 0.7 & 25 & 8 & $\begin{array}{l}1072.32 \\
\pm \\
109.29\end{array}$ & $\begin{array}{l}31.31 \pm \\
2.86\end{array}$ & $\begin{array}{l}2.73 \pm \\
1.27\end{array}$ & $\begin{array}{l}19.33 \pm \\
1.52\end{array}$ \\
\hline 16 & 0.4 & 0.4 & 20 & 10 & $\begin{array}{l}603.75 \pm \\
61.47\end{array}$ & $\begin{array}{l}42.9 \pm \\
3.18\end{array}$ & $\begin{array}{l}2.83 \pm \\
0.55\end{array}$ & $\begin{array}{l}48.66 \pm \\
5.03\end{array}$ \\
\hline 17 & 0.2 & 0.4 & 20 & 6 & $\begin{array}{l}1562.55 \\
\pm \\
362.87\end{array}$ & $\begin{array}{l}26.06 \pm \\
2.17\end{array}$ & $\begin{array}{l}3.23 \pm \\
1.28\end{array}$ & $\begin{array}{l}29 \pm \\
7.81\end{array}$ \\
\hline 18 & 0.4 & 1 & 30 & 6 & $\begin{array}{l}924.72 \pm \\
259.67\end{array}$ & $\begin{array}{l}49.03 \pm \\
4.14\end{array}$ & $\begin{array}{l}2.8 \pm \\
1.21\end{array}$ & $\begin{array}{l}37.33 \pm \\
5.5\end{array}$ \\
\hline 19 & 0.4 & 1 & 20 & 6 & $\begin{array}{l}855.15 \pm \\
80.43\end{array}$ & $\begin{array}{l}25.95 \pm \\
7.49\end{array}$ & $\begin{array}{l}2.3 \pm \\
1.08\end{array}$ & $\begin{array}{l}165 \pm \\
15\end{array}$ \\
\hline 20 & 0.3 & 0.7 & 30 & 8 & $\begin{array}{l}1101.76 \\
\pm \\
385.33\end{array}$ & $\begin{array}{l}50.47 \pm \\
6.78\end{array}$ & $\begin{array}{l}1.56 \pm \\
0.152\end{array}$ & $\begin{array}{l}26 \pm \\
10.14\end{array}$ \\
\hline 21 & 0.3 & 0.7 & 20 & 8 & $\begin{array}{l}868.37 \pm \\
230.92\end{array}$ & $\begin{array}{l}49.21 \pm \\
19.56\end{array}$ & $\begin{array}{l}2.83 \pm \\
0.66\end{array}$ & $\begin{array}{l}32.66 \pm \\
5.13\end{array}$ \\
\hline
\end{tabular}

The SEM images revealed the bead-free fibers with well-embedded drug in the polymer fibers. The diameter distribution of fibers was confirmed that except for samples $2,12,15,17$, and 20, other samples were in the nanometer scale (Fig. 1 and Table 1).

The relationship between variables and the average diameter of fibers in the various formulations was analyzed and the $3 \mathrm{D}$ response plots were obtained according to Fig. 2A quadratic equation model predicted the average diameter of fibers (Y) as follows (Eq. 2):

$Y$ (average fiber diameter $)=+9579.85738-24681.60350 A-1536.21892 B-369.48738 C+38.31252 D+6113.31083$ $A C+15.84579 C D+19923.60236 A^{2}(2)$

In this equation, A, B, C, D are acetone content, flow rate, \% organic phase, and CUR concentration, respectively. The value of $\mathrm{R}^{2}$ (linear regression coefficient) of fiber diameter was determined as 0.9875 . as maintained by the predicted and observed fiber diameter, the model was revealed to deliver an acceptable and significant performance. On the basis of ANOVA and $95 \%$ confidence intervals, the effects of these models were obtained to be statistically significant ( $p$-value < 0.05). It should be noticed that even though $p$-value $>0.05$ (supplementary Table $s 1$ ) for the linear terms but owing to Hierarchy rule in which the $p$-value $<0.05$ for the higher order (interaction or quadratic) of this variable 29,30 . The results of $p$-value indicted that the total independent parameters have strong effect on the fiber diameters.

According to the 3D plot (Fig. 2A), the diameter of the fibers depends on the flow rate and the amount of acetone. Higher flow rate in higher acetone content increased the fiber diameter. However, increasing the flow rate in lower acetone content 
has contradictory effect on diameter. Fridrikh et al. reported that the lowest fiber diameter is observed at lower flow rates

${ }^{31}$. At low flow rates, a small volume ejecting of solution from nozzle creating a short diameter jet which eventually elongated and form a smaller diameter fiber. On the contrary, at high flow rate in constant voltage, the additional amount of the solution is ejected and the jet cannot be fully stretched out due to the insufficiently charged ions, and the fiber emerges with the thicker diameter ${ }^{32}$. In lower acetone contents, it is thought that decreasing the acetone may cause higher viscosity of the electrospinning solution, which in turn, prevents the fibers from being stretched and reduced in diameter. Figure 2B shows that CUR concentration has an increasing effect on the fiber diameter, which may attributed to the increasing drug content of the fibers. Furthermore, by increasing the organic phase \%, the fiber diameter is decreased (Fig. 2B). By increasing the organic phase, the solubility of CUR was enhanced in the organic phase and consequently forms a more stable emulsion within the aqueous phase, resulting in a smaller diameter fiber.

\section{Correlation between process parameters and drug entrapment}

The drug content of the samples was obtained in comparison with the theoretical value (Table 1). The relationship between main variables and drug content in the samples was confirmed by the ANOVA analysis and 3D plot according to Eq. 3 and Fig. 3.

$\mathrm{Y}($ drug Entrapment $)=+169.33730+257.26672 \mathrm{~A}-18.50821 \mathrm{C}+10.98250 \mathrm{AC}-7.83958 \mathrm{CD}+0.403939 \mathrm{C}^{2}(3)$

The value of $\mathrm{R}^{2}$ of loading content was obtained as 0.9674 . The $p$-value of the effect of flow rate on the entrapment $\%$ was not significant and showed a little effect on it (Table S1).

Based on the absorption spectrum of CUR solution, the maximum peak was obtained at $419 \mathrm{~nm}\left(\lambda_{419}\right)^{33}$ while the maximum peak of the PVPK90 was observed at $208.4 \mathrm{~nm}^{34}$ which is shown a non-interference spectrum. The results of drug content and its 3D diagrams in Design Expert showed that at high levels of acetone, the increase of flow rate causes the increase of the loading content and vice versa (Fig. 2C). This phenomenon can be due to the diameter of the fibers. Since in the high flow rate and acetone, an increase in the diameter of the fibers was showed. The ticker fibers have a great ability to drug loading and reduced the risk of drug loss during the process ${ }^{35}$. Figure 2D shows that the higher levels of CUR $(10 \mathrm{mg} / \mathrm{mL})$ in lower organic phase $(20 \%)$ has an increasing effect on the CUR entrapment. Vice versa, in the lower levels of CUR $(6 \mathrm{mg} / \mathrm{mL})$ in higher organic phase (30\%) has an increasing effect on the CUR entrapment. This phenomenon arise from equal amount of CUR in both $20 \%$ and $30 \%$ organic phase.

\section{Correlation between processing parameters and wetting and disintegration times}

The results of wetting and disintegration tests are shown in Table S1. The effect of main variables on the wetting and disintegration pattern of samples was determined by Design Expert software and mathematical equation (Eqs. 4-5). Figure 3 is shown the 3D plot of wetting and disintegration time as a function of the main variable.

$Y($ Wetting time $)=+2.51530-44.00828 \mathrm{~A}+3.45156 \mathrm{~B}+1.22369 \mathrm{C}-2.19909 \mathrm{D}-16.87500 \mathrm{~A} \times \mathrm{B}+0.462500 \mathrm{~A} \times \mathrm{C}+$ $4.03125 \mathrm{~A} \times \mathrm{D}-0.025901 \mathrm{C}^{2}(4)$

$Y($ Disintegration time $)=+7.58665+646.62160 A+426.86146 B+3.07586 C-54.33780 D-18.05250 A \times C-14.26083$ $B \times C-7.21875 B \times D-41.89792 C \times D+220.08705 B^{2}(5)$

The value of $\mathrm{R}^{2}$ of wetting and disintegration time was obtained as 0.9687 and 0.9587 , respectively. The results of $p$-value indicted that the total independent parameters have strong effect on the wetting and disintegration time. 
The wetting test showed that in low levels of acetone, the increasing the flow rate increased the wetting time. Furthermore, the disintegration test showed a reduction in disintegration time resulting from the decrease of flow rate. These findings are well related to the increased fiber thickness in high flow rate and high acetone condition, which needs more time to complete wetting and disintegration time of fibers. The 3D plots of both wetting and disintegration tests showed that with increasing the CUR concentration in higher organic phase \%, despite the increase of the diameter of the fibers, the wetting and disintegration time decreased. It may be related to the porosity of CUR-loaded fibers which facilitating the fibers wetting and disintegration. However it needs further evaluations to be confirmed.

\section{Choosing the optimal formulation}

Using Design-Expert software, the optimized formulation was selected based on the optimization criteria as minimum diameter of nanofibers, the maximum percentage entrapment, minimum disintegration time and minimum wetting time. Further tests were performed on the selected formulation. According to the desirability graph (supplementary Fig. S1), formulation containing $6 \mathrm{mg} / \mathrm{mL}$ CUR, $30 \%$ organic phase, $0.3 \%$ acetone content with a flow rate of $0.75 \mathrm{~mL} / \mathrm{h}$, has the highest desirability (1.0) and was chosen as the optimum formulation.

\section{FTIR analysis}

FTIR spectroscopy is often applied as a proper tool to determine the specific functional groups or chemical bonds of components ${ }^{36}$. FTIR analysis of the nanofiber sample indicated the presence of the main functional groups of CUR and PVP. In the spectrum of CUR (supplementary Fig. S2), the band at $1594.4 \mathrm{~cm}^{-1}$ indicates the overlap of alkene vibrations $(C=C)$ and carbonyl group $(C=0)$. The peak of $3503.3 \mathrm{~cm}^{-1}$ was related to the $0-\mathrm{H}$ group and the peak of $1506.7 \mathrm{~cm}^{-1}$ is related to a mixture of carbonyl bonds in $\mathrm{CC}-\mathrm{C}, \mathrm{CC}-\mathrm{O}$, and $\mathrm{CC}-\mathrm{H}$ aromatic bonds and keto-enol deformation. Similar results have been reported in a study by Ismail et al. ${ }^{37}$. The IR spectrum of PVPK90 (Fig. S2 B) showed a peak at $1428.8 \mathrm{~cm}^{-1}$, which is related to the $\mathrm{C}-\mathrm{N}$ bond. The $1667.4 \mathrm{~cm}^{-1}$ peak is corresponded to the $\mathrm{C}=\mathrm{O}$ bond of the pyrrolidone ring. The peak of $2953 \mathrm{~cm}^{-1}$ is related to $\mathrm{CH}_{2}$ bending vibrations. The broad absorption at $3435.1 \mathrm{~cm}^{-1}$ has contributions from N-H bending vibrations. A same IR spectra for CUR and PVP was observed in a CUR-loaded PVP nanofiber ${ }^{38}$. Baganiz et al. reported the same IR spectra for PVP in carboxylated PVP conjugated with interleukin-10 ${ }^{39}$. The spectrum of PVPcontaining fiber (Fig. S2 C) was almost similar to that of PVP powder, indicating that the nature and chemical structure of PVPK90 did not change in the electrospinning process. In the selected CUR-loaded fiber spectrum (Fig. S2 D), the peak of $3444.9 \mathrm{~cm}^{-1}$ is stronger than the PVP peak in the same range as well as a phenolic $\mathrm{OH}$ bending vibration of CUR is seen in this range demonstrating the hydrogen bond between CUR and PVP. The peak at $1667.4 \mathrm{~cm}^{-1}$ was also strong in the selected fiber in comparison with PVP.

\section{DSC analysis}

DSC analysis showed a peak at $168.3^{\circ} \mathrm{C}$ for CUR confirming its melting point (Fig. 4A). Hani et al. showed a similar melting point for CUR in a complex of CUR and hydroxypropyl-cyclodextrin ${ }^{40}$. In PVP, a peak at $\sim 75^{\circ} \mathrm{C}$ was observed which was ascribed to the water loss (Fig. 4B). The water in the PVP acts as a plasticizer and shifts the melting point of the polymer to the left, and can be attributed to cover the melting point peak of PVP. In a study performed on ketoconazole-coated PVP polymer, a peak at $128^{\circ} \mathrm{C}$ was reported in DSC analysis ${ }^{41}$. In another study, the DSC analysis of carbamazepine/PVP polymer has revealed a peak in the range of $75-140^{\circ} \mathrm{C}$ which can be associated with water release from this hydrophilic polymer ${ }^{42}$. In PVP-containing fiber, a peak was observed as similar as PVP powder, demonstrating no change in the melting point of PVP polymer in the electrospinning process (Fig. 4C). In the DSC of selective fiber (CURloaded fibers), no peak was observed at $168.3^{\circ} \mathrm{C}$ (Fig. 4D) confirming the creation of an amorphous form of drug which also enhanced its water solubility.

\section{$\mathrm{XRD}$ analysis}


The X-ray spectrum in CUR powder showed that the drug has both amorphous and crystalline (with a higher proportion) properties (supplementary Fig. S3A). Previously, XRD analysis on CUR at $2 \theta$ values was verified the main peak of CUR nanocrystals in the same range ${ }^{43}$. In the X-ray spectra of PVP (supplementary Fig. S3B), two broad peaks were exhibited at around $11-13^{\circ}$ and $21^{\circ}$, representing amorphous properties of PVPK90 which was similar to obtained X-ray spectra of PVP by previous reports $41,44,45$. The presence of PVP peak in diffractograms of PVPK90 nanofibers, confirming the presence of PVP (supplementary Fig. S3C). In the selected fiber (supplementary Fig. S3D), the CUR peaks were lost, indicating the complete conversion of the drug to the amorphous form, which has a great role in improving the fiber dissolution. X-ray results can be confirmed by the DSC analyses.

\section{Fluorescence and TEM imaging}

The fluorescence microscopy image of selected nanofiber investigated the presence of CUR in the structure of nanofibers (Fig. 5A and B). TEM image of selected nanofiber at magnification of 1000 (Fig. 5C) and 12930 (Fig. 5D) confirmed the core-shell and uniform properties of nanofibers. In a similar study, the uniform and bead free CUR-loaded nanofibers were indicated by TEM ${ }^{10}$.

\section{Tensile test (Elongation \% and E-Modulus)}

The results of the tensile test were evaluated using a force-displacement diagram based on elastic modulus variable (supplementary Fig. S4). The Elongation and E-Modulus were obtained $17.2 \pm 0.82 \%$ and $5.563 \pm 1.428 \mathrm{MPa}$, respectively.

\section{In vitro CUR release pattern}

The release test results showed that the whole content of CUR released from the selected fiber within 30 sec in PBS while the dissolving of CUR powder was much slower, since only about $40 \%$ of the CUR was dissolved within 60 min (Fig. 6). In this study, the release patterns of CUR from fibers arise from two phases including the initial burst release which was similar to previous reported CUR release profile form electrospun fibers since CUR was completely released from the fiber within $1 \mathrm{~h}^{46}$. It was also reported the release of pure CUR powder less than $15 \%$ within $1 \mathrm{~h}^{47}$. The rapid release can be owing to high surface area of electrospun fibers enhancing the release rate of CUR ${ }^{48}$, fibers diameter, and intermolecular interaction between CUR and PVP ${ }^{46}$. Moreover, it is demonstrated that the porosity of the fiber influence on the release rate of a drug incorporated within an electrospun fiber 49 .

\section{Conclusion}

In this study, PVPK90 core-shell nanofibers containing CUR were prepared to increase the amount and rate of drug release using the electrospinning method and its properties were evaluated. The effect of different parameters on PVPK90-based fiber formation was investigated and four main parameters of flow rate, percentage of the organic phase, amount of drug and acetone were considered as independent variables and 21 formulations were determined by CCD. After characterization and optimization of nanofibers, the formulation containing $6 \mathrm{mg} / \mathrm{ml}$ CUR and $30 \%$ organic phase with $0.4 \mathrm{~mL}$ acetone and a flow rate of $1 \mathrm{~mL} / \mathrm{h}$ was selected. The SEM and TEM showed bead-free core-shell uniform fibers at the nanometer scale in the selected formulation. The tensile test showed that the selected formulation has good flexibility. The drug entrapment in the nanofiber was obtained about $50 \%$. Wetting and disintegrating times were also short and acceptable in the selected formulation. The drug release pattern showed a rapid release of the drug matching with our research purposes.

\section{Materials And Methods \\ Materials}


PVPK90 (MW = 360 kDa) was purchased from Fluka Chemie AG (Switzerland). Tween 80 and Span 80 (sorbitan oleate) were obtained from Sterling (India) and Daejung chemicals and metals (South Korea), respectively. N-butyl acetate was purchased from Merck and CUR was obtained from Sami Labs Limited (Bengaluru, Karnataka, India). All solutions were prepared with deionized water. All chemicals and solvents were of analytical grade.

\section{Experimental design for preparation of the formulations}

To investigate the nanofiber formulations, a response surface method (RSM) based on central composite design (CCD) was modeled using the Design-Expert software (version 10.0.4). Table 2 shows the main four processing parameters including acetone content, flow rate $(\mathrm{mL} / \mathrm{h})$, \% organic phase, and CUR concentration $(\mathrm{mg} / \mathrm{mL})$ in the formulation in three levels which were used for the formulation design (Table 1). The quadratic model was predicted based on experimental data (Eq. (1)).

Table 2

Range of four independent variables with three levels based on the central composite design

\begin{tabular}{|lllll|}
\hline \multirow{2}{*}{ Levels } & \multicolumn{4}{l}{ Independent variables } \\
\cline { 2 - 4 } & Acetone $(\mathrm{mL})$ & $\begin{array}{l}\text { Flow rate } \\
(\mathrm{mL} / \mathrm{h})\end{array}$ & Organic phase $(\%)$ & Curcumin concentration $(\mathrm{mg} / \mathrm{mL})$ \\
\hline+1 & 0.4 & 1 & 30 & 10 \\
\hline 0 & 0.3 & 0.7 & 25 & 8 \\
\hline-1 & 0.2 & 0.4 & 20 & 6 \\
\hline
\end{tabular}

$Y=\beta_{0}+\sum \beta j \square X i+\sum \beta j j \square j^{2}+\sum \beta j k \square X j \square X k(1)$

where $Y=$ properties of the nanofiber, $\beta o=$ the intercept, $\beta j=$ the linear coefficients, $\beta j j=$ the squared coefficients, $\beta j k=$ the interaction coefficients, and $\mathrm{Xi}, \mathrm{Xj}^{2}, \mathrm{Xj}$. $\mathrm{Xk}=$ the levels of the respective independent parameters.

Using Design-Expert software, the relation between main variables $\left(\mathrm{X}_{1}=\mathrm{A}\right.$ : acetone content $\mathrm{X}_{2}=\mathrm{B}$ : flow rate, $\mathrm{X}_{3}=\mathrm{C}$ : \% organic phase, and $\mathrm{X}_{4}=\mathrm{D}$ : CUR concentration) and nanofiber properties including nanofiber diameter, entrapment percentage, wetting and disintegration time was analyzed.

Table 2

\section{Preparation of nanoemulsion}

For preparing nanofibers loaded with CUR, a nanoemulsion containing PVPK90 in water with a concentration of $25 \mathrm{wt} \%$ as an aqueous phase, and a mixture of two emulsifiers Tween 80 and Span 80 at a ratio of 7:3 at the concentration of 0.5 $w t \%$ as an optimal emulsifier system, was used for emulsion formation. The organic phase was prepared by dissolving CUR (according to Table 1) in N-buthyl acetate as a volatile organic solvent by adding different amounts of acetone as cosolvent. The organic phase was slowly supplemented dropwise to the aqueous phase. The coarse O/W emulsion was mixed with a magnetic stirrer for $15 \mathrm{~min}$ followed by bath sonication for $5 \mathrm{~min}$ to obtain a clear nanoemulsion.

\section{Electrospinning process}

Electrospinning was performed at $35^{\circ} \mathrm{C}$ using a uniaxial electrospinning device equipped with a power supply with a voltage of $0-35 \mathrm{kV}$. The collector was a rotating cylinder covered with aluminum foil. The nanoemulsion was supplied by a $5 \mathrm{~mL}$ syringe pump with a flow rate based on Table 1. A 21G steel needle was used as the nozzle with a modified flow rate and the distance between the nozzle and the collector was adjusted to $180 \mathrm{~mm}$. The rotation speed of the cylinder was set to 170-190 rpm. Finally, the produced nanofibers were removed from the collector and preserved for further tests. 


\section{Characterization of the nanofibers \\ Determination of CUR loading in nanofibers (Entrapment \%)}

$2 \mathrm{mg}$ of the nanofibers was dissolved in $4 \mathrm{~mL}$ ethanol $96 \%$ and the absorbance was calculated at $419 \mathrm{~nm}$ (the maximum peak of CUR absorbance) in triplicates. The percentage of drug loading was determined using the standard curve and compared with the theoretical drug level.

\section{Wetting and disintegration time of nanofibers}

The test specimens $(2 \times 2 \mathrm{~cm})$ of nanofibers were cut. For the wetting test, the samples were placed on filter papers which were initially wetted with PBS buffer $(\mathrm{pH}$ 6.8) and for the assessment of disintegration time, the samples were put in $5 \mathrm{~mL}$ PBS buffer ( $\mathrm{pH} 6.8)$ mixing with a magnetic stirrer for $15 \mathrm{~min}$ at $600 \mathrm{rpm}$. The time of complete wetting and dissolving of each nanofiber specimen was captured and determined using the Galaxy Note 8 smartphone camera at a frame rate of 240 frames/second.

\section{Scanning electron microscopy (SEM) imaging}

The morphology and diameter of nanofibers were investigated by SEM. The nanofibers were coated with a mixture of gold and palladium (Au/Pd) under vacuum and imaged by VP 1450 electron microscope at 1000, 5000, 10000, and 20000 magnifications. The fiber diameter was also determined using Image J 1/50i software.

\section{Differential scanning calorimetry (DSC)}

The thermal behavior of CUR, PVPK90, and PVP nanofibers, nanofibers loaded with CUR was performed by DSC 822e (Mettler Toledo, Switzerland) at the temperature range of $35-350^{\circ} \mathrm{C}$ with a heating rate of $10^{\circ} \mathrm{C} /$ minute under nitrogen atmosphere.

\section{X-ray diffraction (XRD)}

XRD analysis was carried out for CUR, PVPK90, and PVP nanofibers, nanofibers loaded with CUR in the range of $2 \theta$ and zero temperature with EXPLORER model XRD (GNR Company, Italy) which was set to $40 \mathrm{KV}$ voltage and $30 \mathrm{~mA}$ current. Fourier Transform Infrared Spectroscopy (FTIR)

The FTIR spectra of CUR, PVPK90, and PVP nanofibers, nanofibers loaded with CUR were performed by Spectrum two FTIR spectrometer (Perkin Elmer, USA) in the range of $400-4000 \mathrm{~cm}^{-1}$ with a resolution of $8 \mathrm{~cm}^{-1}$.

\section{Fluorescence and Transmission electron microscopy (TEM) imaging}

Fluorescence and TEM microscopy were used to investigate the formation of core-shell structure and the presence of CUR in the structure of nanofibers. For TEM, the CUR-loaded nanofibers were fixed on a grid of copper coated with carbon film and the images were taken at 600, 2156, 6000, 10000, 12930, and 27800 magnifications.

\section{Tensile strength of nanofibers}

The mat stripe-shaped specimen of nanofibers with a dimension of $4 \times 1 \mathrm{~cm}$ with a certain thickness was stuck to the $4 \times$ $4 \mathrm{~cm}$ paper frames by an adhesive tape. The tensile tests were carried out by the Hounsfield H50SK material testing machine equipped with a $1 \mathrm{kN}$ load cell, at the strain rate of $5 \mathrm{~mm} / \mathrm{min}$. The fiber was gradually stretched and the related displacement and force values were recorded. After the sample was torn, the force-displacement diagram was drawn and the parameters of E-modulus, yield point, and elongation were measured by Qmat software.

In vitro CUR release pattern 
The in vitro release pattern of CUR was accomplished in PBS buffer at pH $6.8 \pm 0.2 .10 \mathrm{mg}$ CUR-loaded nanofibers were suspended in $10 \mathrm{~mL}$ PBS buffer and incubated at $37^{\circ} \mathrm{C}$ with shaking at a speed of $50 \mathrm{rpm}$. For estimation of the released CUR, the samples were taken at 0.5, 1, 2, 5, 15, 30, and 60 min and mixed with ethanol $96 \%$ to measure the released CUR spectrophotometry at a wavelength of $419 \mathrm{~nm}$. At predetermined time intervals, the sampled dissolution medium was replaced by the same amount of fresh buffer. The release profile of CUR in the same buffer was obtained as control.

\section{Statistical analysis}

The data were analyzed by one-way ANOVA analysis followed by the Tukey-Kramer post-test to determine the significant difference (a P-value less than 0.05). Data are indicated as the mean \pm standard deviation (SD).

\section{Declarations}

\section{Acknowledgments}

The data presented in this report was a part of the Pharm.D. thesis (Grant NO. 961638) supported by Mashhad University of Medical Sciences.

\section{Author contributions}

Hossein Kamali and Parisa Farzadnia contributed to the collection of the data, data analysis, prepared figures and/or tables, wrote the main manuscript, reviewed drafts of the paper. Hossein Kamali. and Mohammadreza Abbaspour. contributed to the data analysis and edited the paper. Jebraeil Movaffagh and Mohammadreza Abbaspour supervised the study, reviewed and edited of the manuscript. Hossein Kamali performed the experimental design analysis. All co-authors contributed to study design and paper preparation, and reviewed drafts of the paper.

\section{Ethics declarations}

All experiments were approved by the Institutional Ethical Committee and Research Advisory Committee of Mashhad University of Medical Sciences guidelines under registration number (IR.MUMS.PHARMACY.REC.1397.002). All experiments were performed in accordance with relevant guidelines and regulations.

\section{Competing interests}

The authors declare no competing interests.

\section{References}

1 Kocaadam, B. \& Şanlier, N. Curcumin, an active component of turmeric (Curcuma longa), and its effects on health. Critical reviews in food science and nutrition 57, 2889-2895 (2017).

2 M Yallapu, M., Jaggi, M. \& C Chauhan, S. Curcumin nanomedicine: a road to cancer therapeutics. Current pharmaceutical design 19, 1994-2010 (2013).

3 Pari, L., Tewas, D. \& Eckel, J. Role of curcumin in health and disease. Archives of physiology and biochemistry 114, 127-149 (2008).

4 Rahmani, A. H., Alsahli, M. A., Aly, S. M., Khan, M. A. \& Aldebasi, Y. H. Role of curcumin in disease prevention and treatment. Advanced biomedical research 7 (2018). 
5 Yallapu, M. M., Nagesh, P. K. B., Jaggi, M. \& Chauhan, S. C. Therapeutic applications of curcumin nanoformulations. The AAPS journal 17, 1341-1356 (2015).

6 Anand, P., Kunnumakkara, A. B., Newman, R. A. \& Aggarwal, B. B. Bioavailability of curcumin: problems and promises. Molecular pharmaceutics 4, 807-818 (2007).

7 Shome, S., Talukdar, A. D., Choudhury, M. D., Bhattacharya, M. K. \& Upadhyaya, H. Curcumin as potential therapeutic natural product: a nanobiotechnological perspective. Journal of Pharmacy and Pharmacology 68, 1481-1500, https://doi.org/10.1111/jphp.12611 (2016).

8 Lee, W.-H. et al. Recent advances in curcumin nanoformulation for cancer therapy. Expert opinion on drug delivery 11, 1183-1201 (2014).

9 Yallapu, M. M., Jaggi, M. \& Chauhan, S. C. Curcumin nanoformulations: a future nanomedicine for cancer. Drug Discovery Today 17, 71-80, https://doi.org/10.1016/j.drudis.2011.09.009 (2012).

10 Raj, S. \& Shankaran, D. R. Curcumin based biocompatible nanofibers for lead ion detection. Sensors and Actuators B: Chemical 226, 318-325, https://doi.org/10.1016/j.snb.2015.12.006 (2016).

11 Ranjbar-Mohammadi, M. \& Bahrami, S. H. Electrospun curcumin loaded poly( $\varepsilon$-caprolactone)/gum tragacanth nanofibers for biomedical application. International Journal of Biological Macromolecules 84, 448-456, https://doi.org/10.1016/j.ijbiomac.2015.12.024 (2016).

12 Fereydouni, N. et al. Curcumin nanofibers for the purpose of wound healing. Journal of Cellular Physiology 234, 5537-5554, https://doi.org/10.1002/jcp.27362 (2019).

13 Gordon, V., Marom, G. \& Magdassi, S. Formation of hydrophilic nanofibers from nanoemulsions through electrospinning. International Journal of Pharmaceutics 478, 172-179, https://doi.org/10.1016/j.ijpharm.2014.11.038 (2015).

14 Bhardwaj, N. \& Kundu, S. C. Electrospinning: A fascinating fiber fabrication technique. Biotechnology Advances 28, 325-347, https://doi.org/10.1016/j.biotechadv.2010.01.004 (2010).

15 Xue, J., Xie, J., Liu, W. \& Xia, Y. Electrospun nanofibers: new concepts, materials, and applications. Accounts of chemical research 50, 1976-1987 (2017).

16 Mutlu, G., Calamak, S., Ulubayram, K. \& Guven, E. Curcumin-loaded electrospun PHBV nanofibers as potential wound-dressing material. Journal of Drug Delivery Science and Technology 43, 185-193, https://doi.org/10.1016/j.jddst.2017.09.017 (2018).

17 Jiang, S. et al. Electrospun nanofiber reinforced composites: a review. Polymer Chemistry 9, 2685-2720, 10.1039/C8PY00378E (2018).

18 Zhang, C., Feng, F. \& Zhang, H. Emulsion electrospinning: Fundamentals, food applications and prospects. Trends in Food Science \& Technology 80, 175-186, https://doi.org/10.1016/j.tifs.2018.08.005 (2018).

19 Wang, C., Tong, S. N., Tse, Y. H. \& Wang, M. in Advanced Materials Research. 118-121 (Trans Tech Publ).

20 Buzgo, M., Mickova, A., Rampichova, M. \& Doupnik, M. in Core-Shell Nanostructures for Drug Delivery and Theranostics (eds Maria Letizia Focarete \& Anna Tampieri) 325-347 (Woodhead Publishing, 2018). 
21 McClellan, P. \& Landis, W. J. Recent applications of coaxial and emulsion electrospinning methods in the field of tissue engineering. BioResearch open access 5, 212-227 (2016).

22 Nikmaram, N. et al. Emulsion-based systems for fabrication of electrospun nanofibers: Food, pharmaceutical and biomedical applications. RSC advances 7, 28951-28964 (2017).

23 Yu, D.-G., Zhang, X.-F., Shen, X.-X., Brandford-White, C. \& Zhu, L.-M. Ultrafine ibuprofen-loaded polyvinylpyrrolidone fiber mats using electrospinning. Polymer Internationa/ 58, 1010-1013, https://doi.org/10.1002/pi.2629 (2009).

24 Koczkur, K. M., Mourdikoudis, S., Polavarapu, L. \& Skrabalak, S. E. Polyvinylpyrrolidone (PVP) in nanoparticle synthesis. Dalton Transactions 44, 17883-17905 (2015).

25 Kurakula, M. \& Rao, G. S. N. K. Pharmaceutical assessment of polyvinylpyrrolidone (PVP): As excipient from conventional to controlled delivery systems with a spotlight on COVID-19 inhibition. Journal of Drug Delivery Science and Technology 60, 102046, https://doi.org/10.1016/j.jddst.2020.102046 (2020).

26 Bühler, V. Polyvinylpyrrolidone excipients for pharmaceuticals: povidone, crospovidone and copovidone. (Springer Science \& Business Media, 2005).

27 Wu, C. S., Senak, L., Bonilla, J. \& Cullen, J. Comparison of relative viscosity measurement of polyvinylpyrrolidone in water by glass capillary viscometer and differential dual-capillary viscometer. Journal of applied polymer science $\mathbf{8 6}$, 1312-1315 (2002).

28 Korycka, P., Mirek, A., Kramek-Romanowska, K., Grzeczkowicz, M. \& Lewińska, D. Effect of electrospinning process variables on the size of polymer fibers and bead-on-string structures established with a 23 factorial design. Beilstein journal of nanotechnology 9, 2466-2478 (2018).

29 Kamali, H., Golmakani, E., Golshan, A., Mohammadi, A. \& Sani, T. A. Optimization of ethanol modified supercritical carbon dioxide on the extract yield and antioxidant activity from Biebersteinia multifida DC. The Journal of Supercritical Fluids 91, 46-52 (2014).

30 Kamali, H., Khodaverdi, E., Hadizadeh, F. \& Ghaziaskar, S. Optimization of phenolic and flavonoid content and antioxidants capacity of pressurized liquid extraction from Dracocephalum kotschyi via circumscribed central composite. The Journal of Supercritical Fluids 107, 307-314 (2016).

31 Fridrikh, S. V., Jian, H. Y., Brenner, M. P. \& Rutledge, G. C. Controlling the fiber diameter during electrospinning. Physical review letters 90, 144502 (2003).

32 Zargham, S., Bazgir, S., Tavakoli, A., Rashidi, A. S. \& Damerchely, R. The effect of flow rate on morphology and deposition area of electrospun nylon 6 nanofiber. Journal of Engineered Fibers and Fabrics 7, 155892501200700414 (2012).

33 Perera, W. et al. Curcumin loaded zinc oxide nanoparticles for activity-enhanced antibacterial and anticancer applications. RSC Advances 10, 30785-30795 (2020).

34 Zaman, A. C. \& Kaya, C. Determination of Quantity of Materials in Suspensions and in Electrophoretic Coatings by UV-Visible Absorption Spectroscopy. Journal of The Electrochemical Society 162, D3109 (2015).

35 Reda, R. I., Wen, M. M. \& El-Kamel, A. H. Ketoprofen-loaded Eudragit electrospun nanofibers for the treatment of oral mucositis. International journal of nanomedicine 12, 2335 (2017). 
37 Ismail, E., Sabry, D., Mahdy, H. \& Khalil, M. Synthesis and Characterization of some Ternary Metal Complexes of Curcumin with 1, 10-phenanthroline and their Anticancer Applications. Journal of Scientific Research 6, 509-519 (2014).

38 Luo, X. \& Lim, L.-T. Curcumin-loaded electrospun nonwoven as a colorimetric indicator for volatile amines. LWT 128, 109493 (2020).

39 Baganizi, D. R., Nyairo, E., Duncan, S. A., Singh, S. R. \& Dennis, V. A. Interleukin-10 conjugation to carboxylated PVPcoated silver nanoparticles for improved stability and therapeutic efficacy. Nanomaterials 7, 165 (2017).

40 Hani, U. et al. Design and Optimization of Curcumin-HPßCD Bioadhesive Vaginal Tablets by 23 Factorial Design: In Vitro and In Vivo Evaluation. Journal of Pharmaceutical Innovation 10, 21-35 (2015).

41 Kumar, P., Mohan, C., Shankar, M. K. U. \& Gulati, M. Physiochemical characterization and release rate studies of soliddispersions of ketoconazole with pluronic f127 and pvp k-30. Iranian journal of pharmaceutical research: IJPR 10, 685 (2011).

42 Abedinoghli, D. et al. Electrosprayed nanosystems of carbamazepine-PVP K30 for enhancing its pharmacologic effects. Iranian journal of pharmaceutical research: IJPR 17, 1431 (2018).

43 Rajasekar, A. Facile synthesis of curcumin nanocrystals and validation of its antioxidant activity against circulatory toxicity in Wistar rats. Journal of nanoscience and nanotechnology 15, 4119-4125 (2015).

44 Abdelrazek, E. M., Abdelghany, A. M., Badr, S. I. \& Morsi, M. A. Structural, optical, morphological and thermal properties of PEO/PVP blend containing different concentrations of biosynthesized Au nanoparticles. Journal of Materials Research and Technology 7, 419-431, https://doi.org/10.1016/j.jmrt.2017.06.009 (2018).

45 Bhuiyan, M., Rahman, M., Rahaman, M., Shajahan, M. \& Dafader, N. Improvement of swelling behaviour of poly (vinyl pyrrolidone) and acrylic acid blend hydrogel prepared by the application of gamma radiation. Organic Chem Curr Res 4, 2161-0401.10001 (2015).

46 Rahma, A., Munir, M. M., Prasetyo, A., Suendo, V. \& Rachmawati, H. Intermolecular interactions and the release pattern of electrospun curcumin-polyvinyl (pyrrolidone) fiber. Biological and Pharmaceutical Bulletin 39, 163-173 (2016).

47 Rachmawati, H., Edityaningrum, C. A. \& Mauludin, R. Molecular inclusion complex of curcumin- $\beta$-cyclodextrin nanoparticle to enhance curcumin skin permeability from hydrophilic matrix gel. Aaps Pharmscitech 14, 1303-1312 (2013).

48 Son, Y. J., Kim, W. J. \& Yoo, H. S. Therapeutic applications of electrospun nanofibers for drug delivery systems. Archives of pharmacal research 37, 69-78 (2014).

49 Park, C.-H., Chung, M.-Y., Unnithan, A. R. \& Kim, C. S. Creation of a functional graded nanobiomembrane using a new electrospinning system for drug release control and an in vitro validation of drug release behavior of the coating membrane. Materials Science and Engineering: C 50, 133-140, https://doi.org/10.1016/j.msec.2015.02.001 (2015).

\section{Figures}



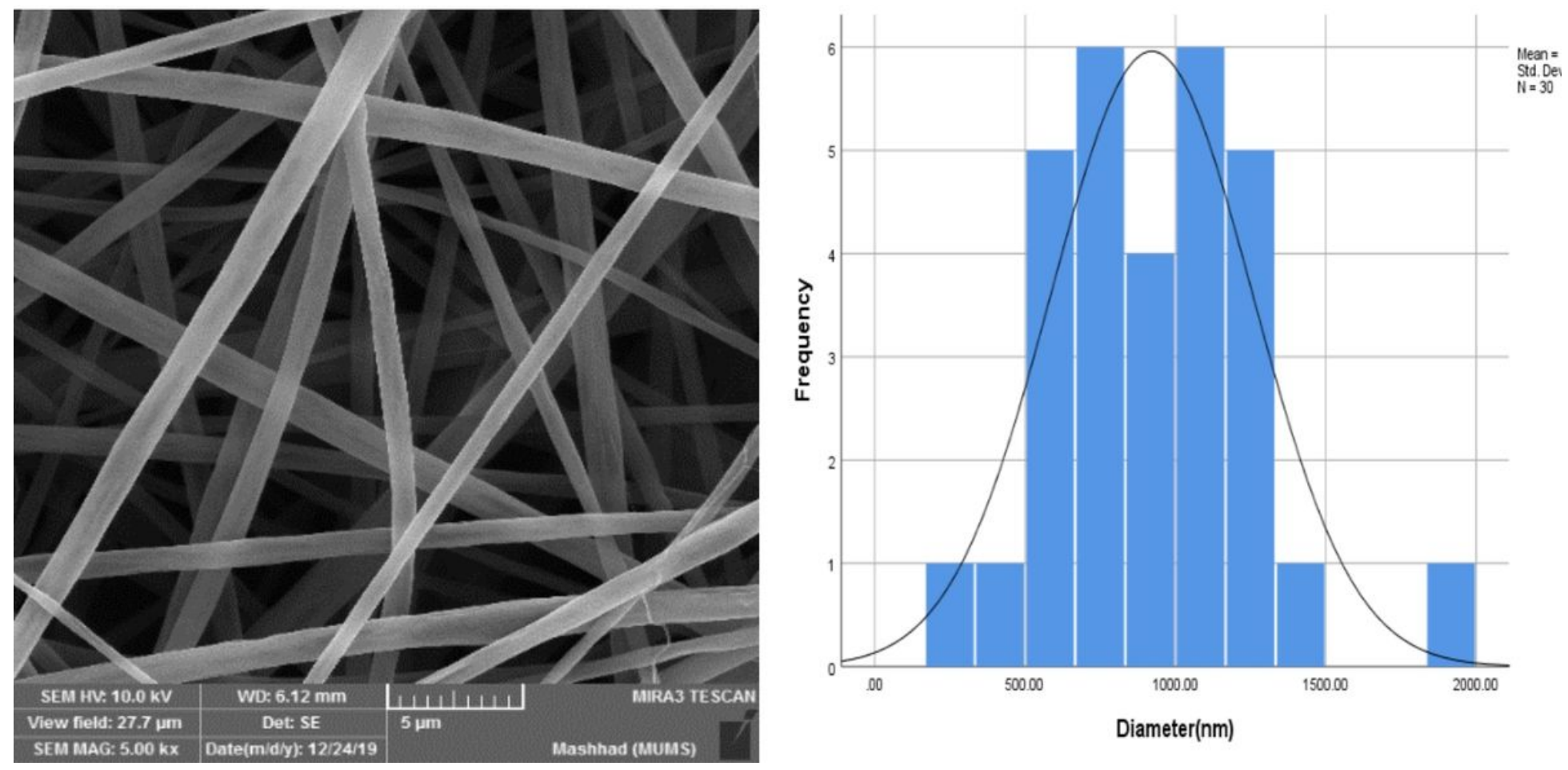

Figure 1

SEM images and diameter distribution of the optimal nanofibers 

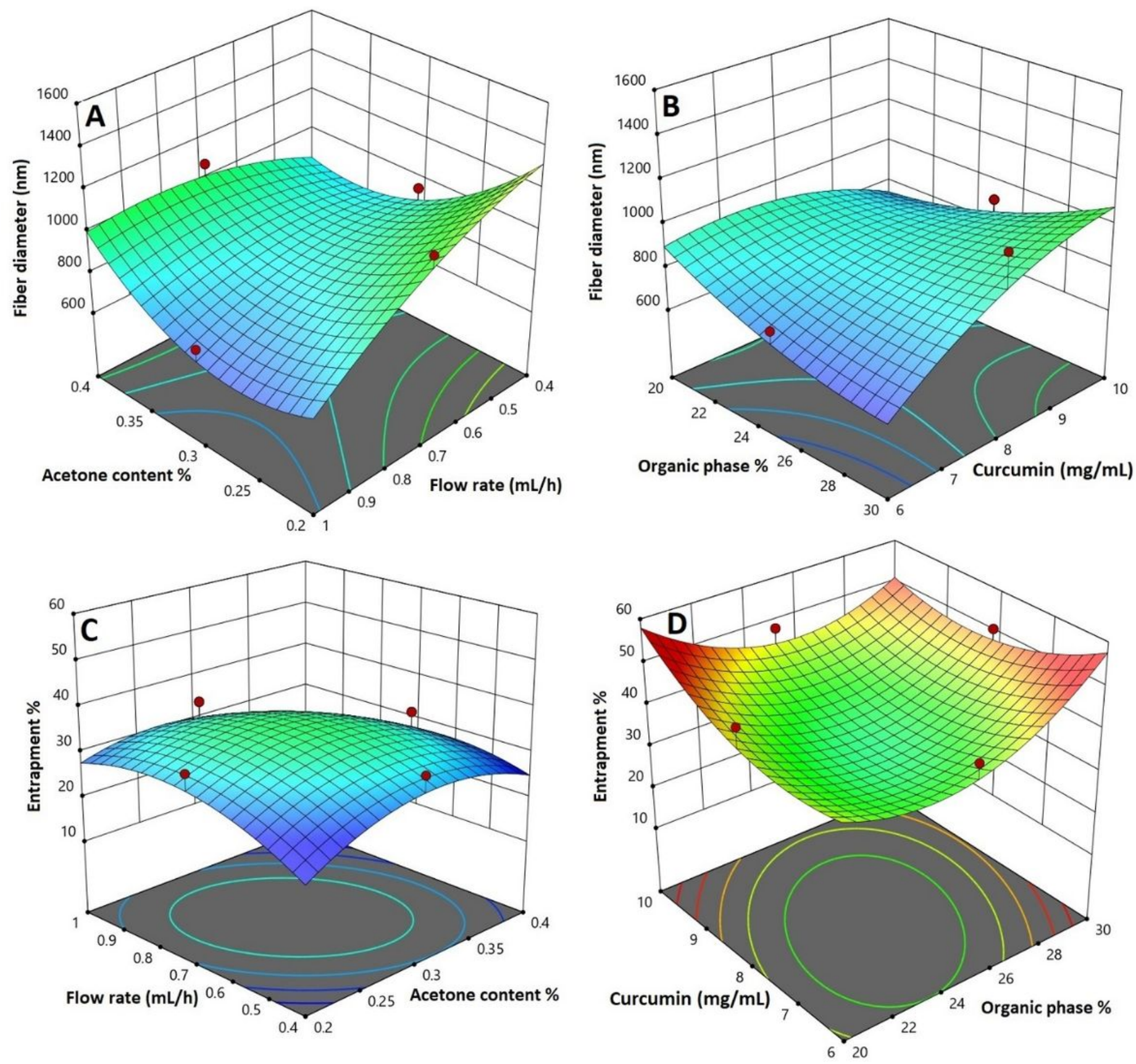

Figure 2

Surface and contour plot of fiber diameter as a function of acetone content and flow rate (A) and as a function of organic phase percent and CUR concentration (B), surface and contour plot of drug entrapment as a function of acetone content and flow rate (C) and as a function of organic phase percent and CUR concentration (D). 

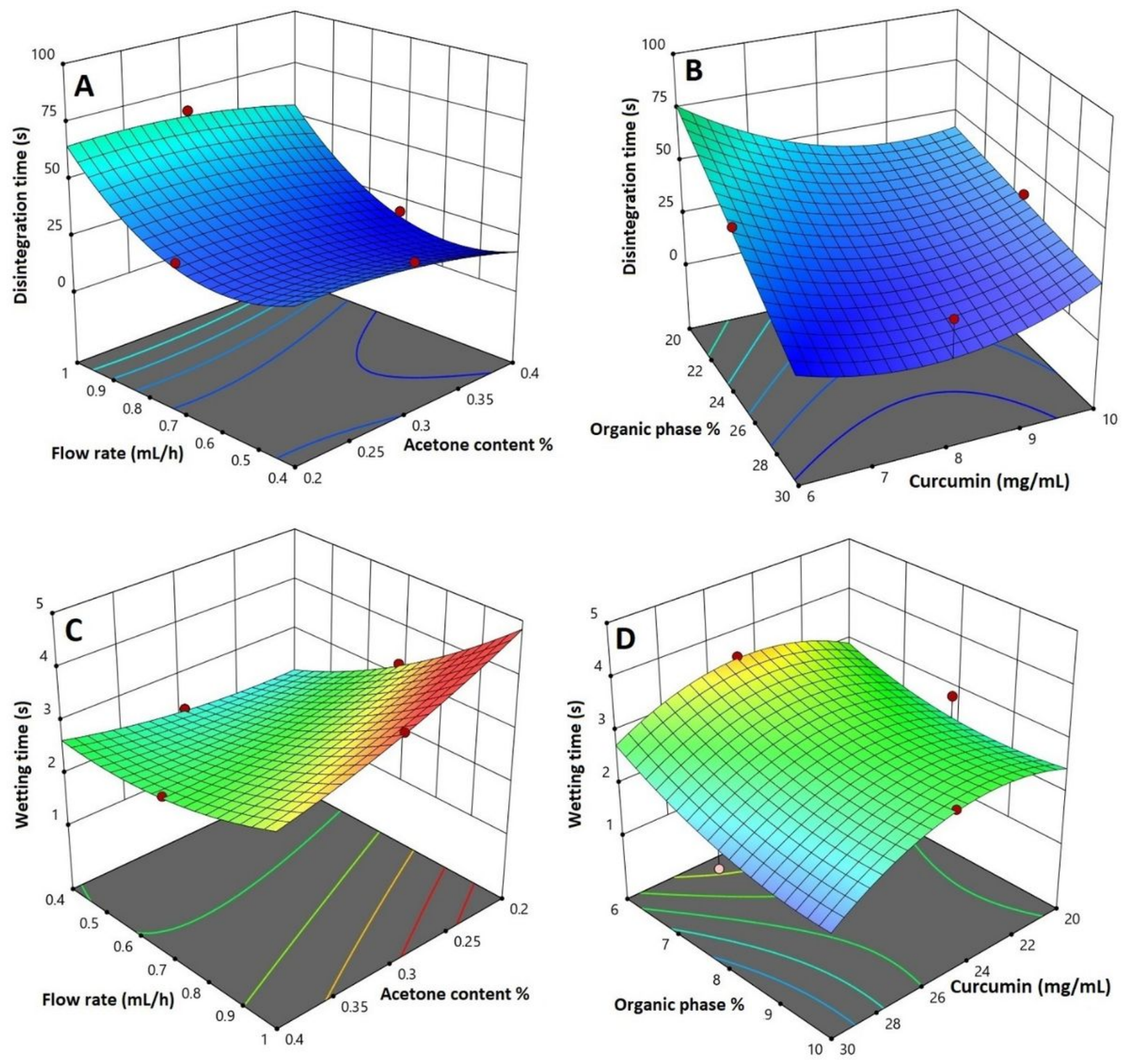

Figure 3

Surface and contour plot of wetting time as a function of acetone content and flow rate (A) and as a function of \% CUR concentration and organic phase percent (B), surface and contour plot of disintegration time as a function of acetone content and flow rate (C) and as a function of \% CUR concentration and organic phase percent (D). 


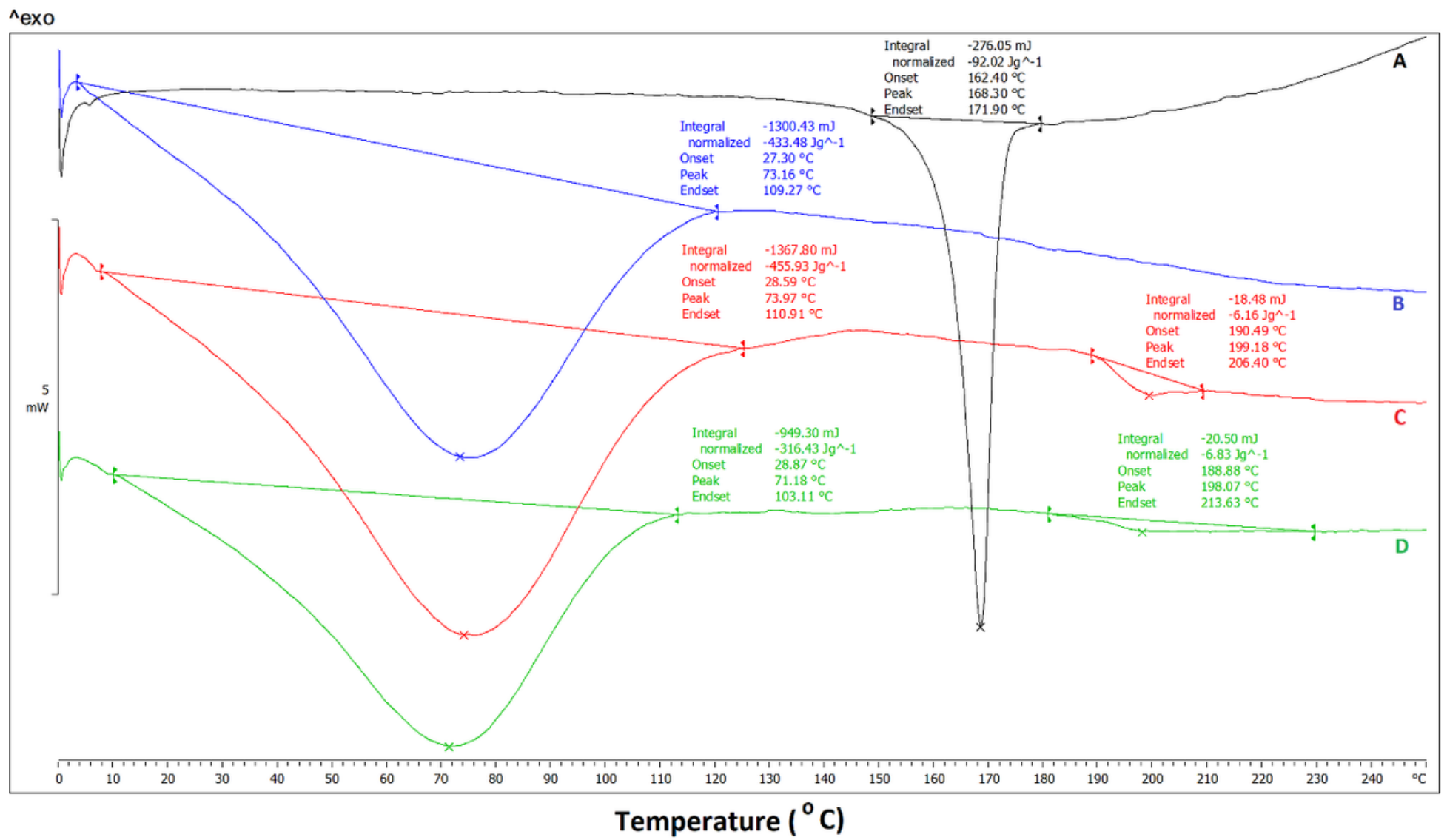

Figure 4

DSC spectra of (A) CUR, (B) PVPK90, (C) PVPK90 nanofibers, and (D) nanofibers loaded with CUR. 


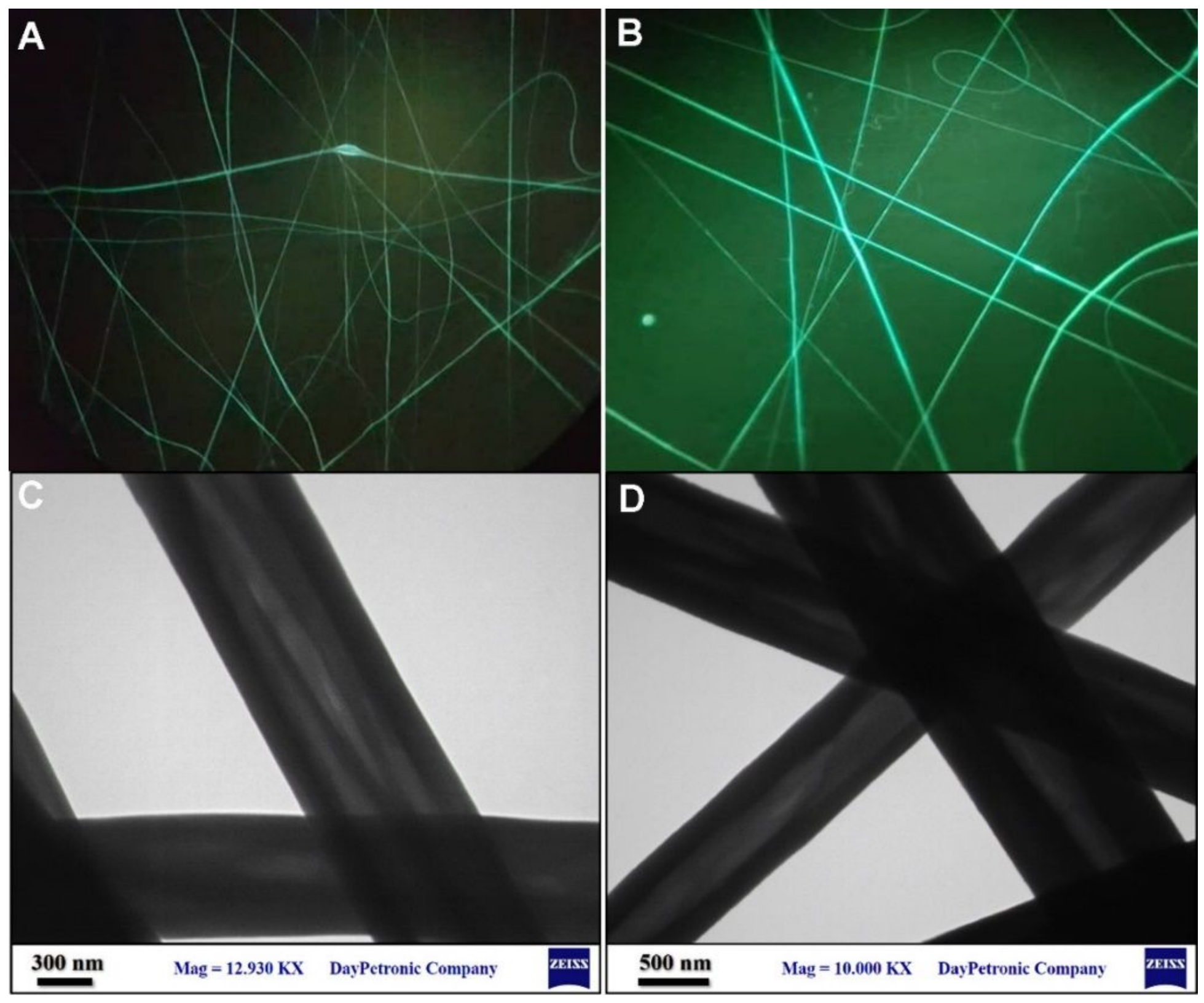

Figure 5

The fluorescence microscopy (A, B) and TEM image of selected nanofiber at 10000 (C) and 12930 (D) magnifications. 


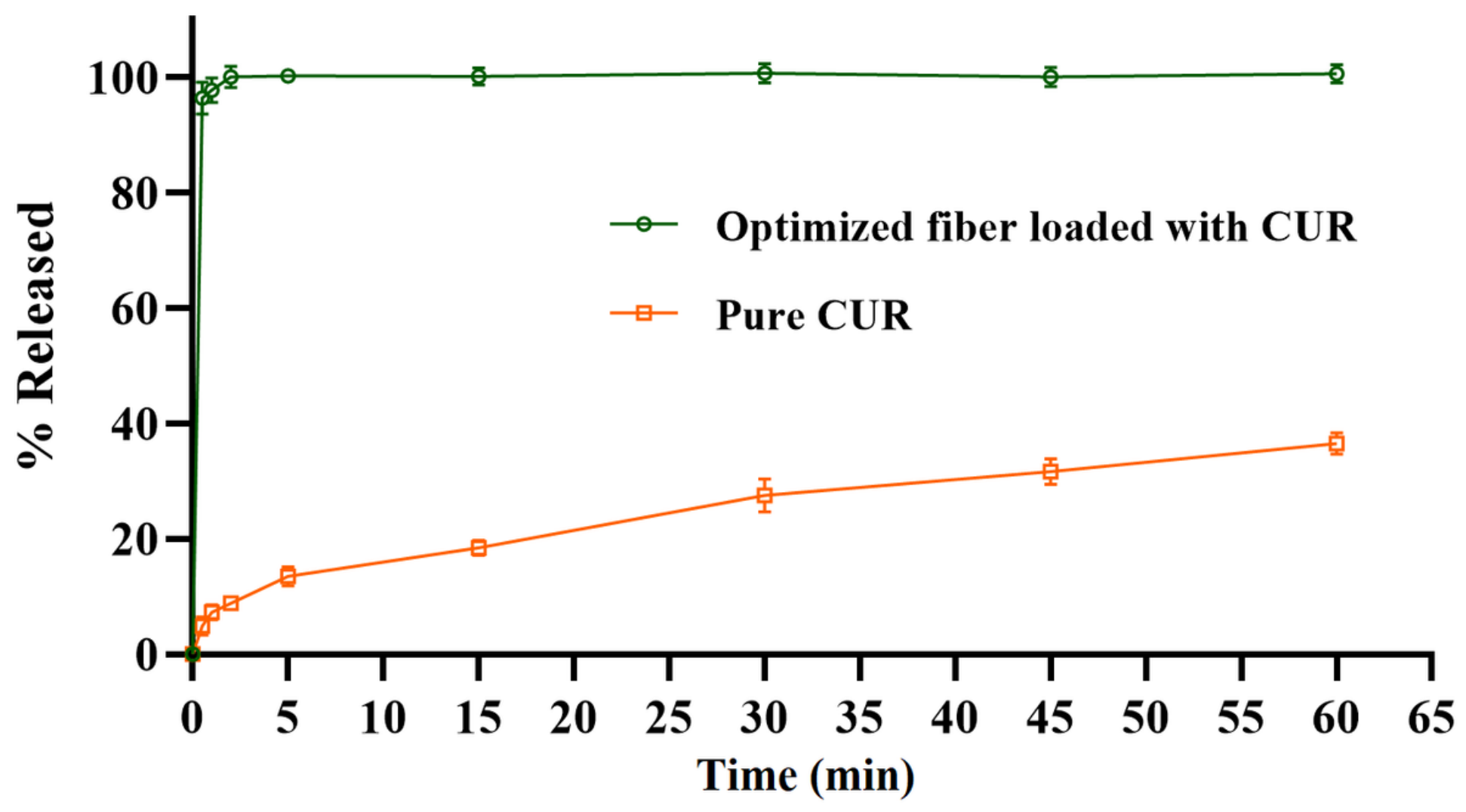

Figure 6

In vitro CUR release profile of nanofibers loaded with CUR in comparison with bulk CUR

\section{Supplementary Files}

This is a list of supplementary files associated with this preprint. Click to download.

- Supplementrydata.pdf 